\title{
Local thermal behaviour of a massive scalar field near a reflecting wall
}

\author{
V.A. De Lorenci, ${ }^{a}$ L.G. Gomes ${ }^{b}$ and E.S. Moreira Jr. ${ }^{b, 1}$ \\ ${ }^{a}$ Instituto de Física e Química, Universidade Federal de Itajubá, \\ Itajubá, MG 37500-903, Brazil \\ ${ }^{b}$ Instituto de Matemática e Computação, Universidade Federal de Itajubá, \\ Itajubá, MG 37500-903, Brazil \\ E-mail: delorenci@unifei.edu.br, lggomes@unifei.edu.br, \\ moreira@unifei.edu.br
}

ABStRact: The mean square fluctuation and the expectation value of the stress-energymomentum tensor of a neutral massive scalar field at finite temperature are determined near an infinite plane Dirichlet wall, and also near an infinite plane Neumann wall. The flat background has an arbitrary number of dimensions and the field is arbitrarily coupled to the vanishing curvature. It is shown that, unlike vacuum contributions, thermal contributions are free from boundary divergences, and that the thermal behaviour of the scalar field near a Dirichlet wall differs considerably from that near a Neumann wall. Far from the wall the study reveals a local version of dimensional reduction, namely, corrections to familiar blackbody expressions are linear in the temperature, with the corresponding coefficients given only in terms of vacuum expectation values in a background with one less dimension. It is shown that such corrections are "classical" (i.e., not dependent on Planck's constant) only if the scalar field is massless. A natural conjecture that arises is that the "local dimensional reduction" is universal since it operates for massless and massive fields alike and regardless of the boundary conditions.

KeYwords: Boundary Quantum Field Theory, Thermal Field Theory

ArXiv EPRINT: 1410.7826

\footnotetext{
${ }^{1}$ Corresponding author.
} 


\section{Contents}

1 Introduction 1

$\begin{array}{llr}2 & \text { Renormalized propagator } & 4\end{array}$

3 Mean square field fluctuation $\quad 5$

$\begin{array}{lll}4 & \text { Stress-energy-momentum tensor } & 7\end{array}$

5 Ultralight scalar radiation $\quad 10$

6 Neumann's boundary condition $\quad 11$

7 Conclusion 13

$\begin{array}{ll}\text { A Finite temperature propagator } & 14\end{array}$

B Alternative expressions for certain averages $\quad 16$

\section{Introduction}

After the recent discovery of the Higgs boson, interest on the physics of massive scalar fields became rather more phenomenological and less academic. As is well known, there are models involving scalar fields in cosmology and high energy physics where a natural protagonist is the Higgs boson (see e.g. ref. [1]). If one adds that primordial hot radiation permeates the universe and that modern colliders recreates the extremely hot conditions just after the big bang, in this scenario it seems pertinent to study the thermal properties of a massive scalar field in great detail.

The tools to study scalar radiation at temperature $T$ in an infinite cavity do not differ from those used in dealing with the familiar electromagnetic blackbody radiation. One can for example identify the scalar radiation in the cavity with an ideal gas of bosons with zero chemical potential, leading to the well known distribution

$$
n_{\mathbf{p}}=\frac{1}{\exp \left\{\beta \sqrt{(p c)^{2}+\left(M c^{2}\right)^{2}}\right\}-1},
$$

where $\beta:=1 / k_{B} T$ as usual. Setting $M=0$ and considering integrations over phase space and momentum, eq. (1.1) yields the familiar Planckian expressions for the energy density $\rho$ and pressure $P$,

$$
\rho=\frac{\pi^{2}}{30} \frac{\left(k_{B} T\right)^{4}}{(\hbar c)^{3}}, \quad P=\frac{\rho}{3}
$$


corresponding to the following uniform and isotropic stress-energy-momentum tensor

$$
\left\langle T^{\mu \nu}\right\rangle=\operatorname{diag}(\rho, P, P, P) .
$$

One might also consider the situation that, although still dealing with an infinite cavity, the interest is in the scalar radiation near, say, a plane wall of the cavity (with the other walls at infinity). Applying local techniques of field theory at finite temperature $T$, assuming that the scalar field is massless, conformally coupled (i.e., with curvature coupling parameter $\xi=1 / 6$ ) and that it satisfies the Dirichlet boundary condition on the wall, Kennedy, Critchley and Dowker [2] have found the following ensemble average near the infinite wall lying on a Cartesian plane,

$$
\left\langle T^{\mu \nu}\right\rangle=\operatorname{diag}\left(\frac{\rho}{9}, P,-\frac{P}{3},-\frac{P}{3}\right),
$$

where $\rho$ and $P$ are those in eq. (1.2) and subleading contributions (that vanish when the distance to the plane wall vanishes) have been neglected. In fact, only the second component in eq. (1.4) - pressure on the wall - is strictly uniform as have been shown by Tadaki and Takagi [3].

The source of apparent conflict between eqs. (1.3) and (1.4) is that by assuming the distribution (1.1) one is ignoring the presence of walls. Boundaries "deform" the vacuum (Casimir-like effects $[4,5])$ and modify ensemble averages. It follows that $\left\langle T^{\mu \nu}\right\rangle$ in eq. (1.3) is correct only if one is considering scalar radiation deep in the bulk, i.e., far away from the walls of the cavity (or, equivalently, at high temperature) $[2,3]$.

By investigating hot radiation near curved boundaries, Balian and Duplantier [6] have shown that similar kind of non trivial local effects also arise with the electromagnetic radiation. In particular they have shown that the energy density presents a non integrable divergence when a curved wall of a perfect conductor is approached. Boundary divergences were also investigated by DeWitt in the classic text [7] concluding that even near a reflecting plane wall the vacuum expectation value $\left\langle T^{\mu \nu}\right\rangle$ of a non conformally coupled scalar field diverges at the wall. In fact, for an arbitrary $\xi$ eq. (1.4) is replaced by [3]

$$
\left\langle T^{\mu \nu}\right\rangle=\operatorname{diag}\left(\rho_{0}+\frac{(1-4 \xi) \rho}{3}, P,-\rho_{0}-(1-4 \xi) P,-\rho_{0}-(1-4 \xi) P\right),
$$

where the vacuum energy density

$$
\rho_{0}:=-\frac{(1-6 \xi) \hbar c}{16 \pi^{2} x^{4}}
$$

carries the non integrable divergence arising when the Dirichlet wall is approached $(x \rightarrow 0)$.

Boundary divergences were interpreted by Deutsch and Candelas [8] as a consequence of oversimplification of real boundaries, and that has at some extend been confirmed along the years by further investigations [9-16]. To keep these divergences under control one can use certain "cut-off procedures" according to which vacuum expectation values such as $\rho_{0}$ in eq. (1.6) hold close, but not that close to the wall. An alternative and interesting way of dealing with boundary divergences is the "renormalization procedure" suggested in ref. [2] 
(see also ref. [17]). Within this approach $\rho_{0}$ in eq. (1.6) hides a $\delta$-function contribution which, after integration over space, gives rise to a surface energy that kills off the divergent contribution in the formal expression for the total vacuum energy, resulting that the latter becomes finite. The "renormalization procedure" was extended to the electromagnetic field in ref. [18], and to deal with more general boundary conditions for the massless scalar field on plane boundaries in ref. [19]. Also worth mentioning is a "cut-off procedure" (possibly related with the "renormalization procedure" just considered) that has been proposed by Ford and Svaiter [20]. It consists of taking into account the quantum nature of the boundary, resulting that the "width" of the boundary's wave packet becomes a regulator parameter.

Investigations on the global thermal behaviour of the scalar field in backgrounds with boundaries go back to the 1970's [21, 22] and since then the subject has been much considered in the literature (see reviews [4,5] and refs. [23-30]), unlike its local thermal behaviour that has been much less studied. Early investigations on the local thermal behaviour of a scalar field near a reflecting wall have been restricted to massless fields $[2,3]$. The present paper extends the study to examine a massive field. In so doing, the philosophy in the work by Brown and Maclay [31] to deal with the electromagnetic field between conducting plates is followed. Namely, the ensemble average of a quantity $\mathcal{A}$ will be written as,

$$
\langle\mathcal{A}\rangle=\langle\mathcal{A}\rangle_{\text {vacuum }}+\langle\mathcal{A}\rangle_{\text {mixed }}+\langle\mathcal{A}\rangle_{\text {thermal }}
$$

where $\langle\mathcal{A}\rangle_{\text {vacuum }}$ is the vacuum expectation value of $\mathcal{A}$ (obtained by setting the temperature equal to zero in eq. (1.7)), $\langle\mathcal{A}\rangle_{\text {thermal }}$ is the blackbody contribution (that obtained by using eq. (1.1)), and $\langle\mathcal{A}\rangle_{\text {mixed }}$ is a "mixed" contribution (with vacuum-thermal nature) that connects the two others such that it vanishes at zero temperature and far away from the reflecting wall.

To arrive to $\langle\mathcal{A}\rangle$ for a massive and arbitrarily coupled scalar field $\phi$, the "pointsplitting" approach $[32,33]$ is applied to the Schwinger "proper time" representation of the Feynman propagator (see e.g. ref. [8]) at finite temperature. The latter is calculated in appendix A for a flat $N$-dimensional spacetime containing an infinite plane wall where $\phi$ satisfies the Dirichlet (or Neumann) boundary condition. In section 2, the Feynman propagator is renormalized giving rise to an expression involving infinite sums of modified Bessel functions of the second kind $K_{\nu}(\mathrm{z})$, where $\nu$ is fixed by the dimensionality $N$ of the spacetime. The ensemble averages $\left\langle\phi^{2}\right\rangle$ and $\left\langle T^{\mu \nu}\right\rangle$ are found and investigated in sections 3 and 4 , respectively. In section 5 , the leading order behaviours of $\left\langle\phi^{2}\right\rangle$ and $\left\langle T^{\mu \nu}\right\rangle$ for an "ultralight" scalar field (i.e., the corresponding massless expressions) are briefly addressed. (It should be pointed out that $\left\langle T^{\mu \nu}\right\rangle$ for hot massless scalar radiation near a reflecting wall has been studied previously $[2,3,34]$; whereas no record was found on $\left\langle\phi^{2}\right\rangle$.) In section 6 , the arguments developed in the previous sections for the Dirichlet boundary condition are extended to consider Neumann's boundary condition, and a parallel between the corresponding thermal behaviours is drawn. Section 7 contains a summary and some remarks on the ensemble averages found in the text, ending with proposals on further study of a local version of the dimensional reduction, noted in this work, which relates vacuum averages in $N-1$ dimensions to (in general "non-classical") corrections to blackbody expressions in 
$N$ dimensions. Appendix B contains alternative expressions that turned out to be useful in the text. (Unless stated otherwise, dimensions are such that $k_{B}=\hbar=c=1$.)

\section{Renormalized propagator}

Consider an infinite cavity in an $N$-dimensional flat spacetime (cf. eq. (A.1)). One of the walls of the $(N-1)$-dimensional cavity coincides with the plane $x=0$, and the other walls are at infinity. A point in the spacetime is labelled by flat coordinates $(t, x, y, z, \cdots)$ where $|x|$ is the distance to the plane wall. Due to the obvious symmetry of the background, $x$ is taken to be non negative in the rest of the text. A neutral scalar field $\phi$ with mass $M$ is assumed to be confined to the cavity and in thermodynamic equilibrium with the walls at temperature $T$. To prevent fluxes through the plane wall, a typical boundary condition used is Dirichlet's which will be taken here. Thus $\phi=0$ at $x=0$. (Neumann's boundary condition is addressed in sections 6 and 7.)

In order to obtain the Feynman propagator $G_{\mathcal{F}}\left(\mathrm{x}, \mathrm{x}^{\prime}\right)$ at finite temperature $T$ one solves

$$
\left(\square_{\mathrm{x}}+M^{2}\right) G_{\mathcal{F}}\left(\mathrm{x}, \mathrm{x}^{\prime}\right)=-\delta\left(\mathrm{x}-\mathrm{x}^{\prime}\right)
$$

observing the usual prescription (see e.g. ref. [35]) of continuing time $t$ to imaginary values, taking it periodic with period $1 / T$. This is done in appendix $\mathrm{A}$, and the result is

$$
\begin{aligned}
G_{\mathcal{F}}\left(\mathrm{x}, \mathrm{x}^{\prime}\right)= & -\frac{i}{(2 \pi)^{N / 2}} M^{\frac{N-2}{2}} \\
& \times \sum_{n=-\infty}^{\infty}\left[\left(-\sigma_{-}\right)^{\frac{2-N}{4}} K_{\frac{N-2}{2}}\left(M \sqrt{-\sigma_{-}}\right)-\left(-\sigma_{+}\right)^{\frac{2-N}{4}} K_{\frac{N-2}{2}}\left(M \sqrt{-\sigma_{+}}\right)\right],
\end{aligned}
$$

where $\sigma_{ \pm}:=\left(t-t^{\prime}-i n / T\right)^{2}-\left(x \pm x^{\prime}\right)^{2}-\left(y-y^{\prime}\right)^{2}-\left(z-z^{\prime}\right)^{2}-\cdots$. Recalling that for small argument and $\nu>0$,

$$
K_{\nu}(\mathrm{z})=2^{\nu-1} \Gamma(\nu) \mathrm{z}^{-\nu}+\cdots,
$$

by setting $M \rightarrow 0$ in eq. (2.2) the massless propagator is recovered [34].

By studying eq. (2.2), one sees that the term corresponding to $n=0$ and involving $\sigma_{-}$ is simply the familiar vacuum propagator in Minkowski spacetime $G_{0}\left(\mathrm{x}, \mathrm{x}^{\prime}\right)$. That corresponding to $n=0$ but now involving $\sigma_{+}$is the vacuum propagator in the presence of the Dirichlet wall $G_{\text {vacuum }}\left(\mathrm{x}, \mathrm{x}^{\prime}\right)$. The first sum $\sum_{n \neq 0}$ in eq. (2.2) yields the thermal propagator in Minkowski spacetime $G_{\text {thermal }}\left(\mathrm{x}, \mathrm{x}^{\prime}\right)$, while the second sum $\sum_{n \neq 0}$ yields a propagator $G_{\text {mixed }}\left(\mathrm{x}, \mathrm{x}^{\prime}\right)$ of a mixed (vacuum-thermal) nature. Therefore eq. (2.2) can be cast as

$$
G_{\mathcal{F}}\left(\mathrm{x}, \mathrm{x}^{\prime}\right)=G_{0}\left(\mathrm{x}, \mathrm{x}^{\prime}\right)+G_{\text {vacuum }}\left(\mathrm{x}, \mathrm{x}^{\prime}\right)+G_{\text {mixed }}\left(\mathrm{x}, \mathrm{x}^{\prime}\right)+G_{\text {thermal }}\left(\mathrm{x}, \mathrm{x}^{\prime}\right) .
$$

Since spacetime is flat one renormalizes by removing the zero temperature Minkowski contribution, resulting the renormalized propagator,

$$
G\left(\mathrm{x}, \mathrm{x}^{\prime}\right)=G_{\text {vacuum }}\left(\mathrm{x}, \mathrm{x}^{\prime}\right)+G_{\text {mixed }}\left(\mathrm{x}, \mathrm{x}^{\prime}\right)+G_{\text {thermal }}\left(\mathrm{x}, \mathrm{x}^{\prime}\right) .
$$


As averages can formally be obtained by linear operations in the renormalized propagator $[32,33]$, the expression for the ensemble average $\langle\mathcal{A}\rangle$ in eq. (1.7) follows from eq. (2.5). Taking into account that for large argument $K_{\nu}(\mathrm{z})$ falls exponentially, the following limits can be readily verified,

$$
\lim _{T \rightarrow 0} G\left(\mathrm{x}, \mathrm{x}^{\prime}\right)=G_{\text {vacuum }}\left(\mathrm{x}, \mathrm{x}^{\prime}\right), \quad \quad \lim _{x, x^{\prime} \rightarrow \infty} G\left(\mathrm{x}, \mathrm{x}^{\prime}\right)=G_{\text {thermal }}\left(\mathrm{x}, \mathrm{x}^{\prime}\right) .
$$

In both these limits $G_{\text {mixed }}\left(\mathrm{x}, \mathrm{x}^{\prime}\right)$ vanishes, and all that still holds if the field is massless with $N>3$. (For $N \leq 3$ when $M=0$, divergences typical of lower dimensions appear [35]. More on that in section 5.)

\section{Mean square field fluctuation}

The ensemble average $\left\langle\phi^{2}\right\rangle$ measures how much the scalar field $\phi$ fluctuates around $\langle\phi\rangle=0$. It is obtained from eq. (2.5) simply by considering $\left\langle\phi^{2}\right\rangle=i G(\mathrm{x}, \mathrm{x})$,

$$
\left\langle\phi^{2}\right\rangle^{(N)}=\left\langle\phi^{2}\right\rangle_{\text {vacuum }}^{(N)}+\left\langle\phi^{2}\right\rangle_{\text {mixed }}^{(N)}+\left\langle\phi^{2}\right\rangle_{\text {thermal }}^{(N)},
$$

where the superscript $(N)$ is not an exponent but simply indicates dimensionality, which will play special role below. The last term in eq. (3.1) is the uniform blackbody contribution,

$$
\left\langle\phi^{2}\right\rangle_{\text {thermal }}^{(N)}=\frac{1}{\pi}\left(\frac{M T}{2 \pi}\right)^{\frac{N-2}{2}} \sum_{n=1}^{\infty} n^{\frac{2-N}{2}} K_{\frac{N-2}{2}}\left(\frac{M n}{T}\right) .
$$

(For a quick check of eq. (3.2) one sets $N=4$ and $M \rightarrow 0$ observing eq. (2.3), then the sum yields $\zeta(2)$ leading to the familiar expression $T^{2} / 12$.) The first term is the vacuum fluctuation,

$$
\left\langle\phi^{2}\right\rangle_{\text {vacuum }}^{(N)}=-\frac{1}{2^{N-1} \pi^{N / 2}}\left(\frac{M}{x}\right)^{\frac{N-2}{2}} K_{\frac{N-2}{2}}(2 M x),
$$

where by setting $N=4$ and $M \rightarrow 0$ one recovers the well known expression $-1 / 16 \pi^{2} x^{2}$ [33]. Equation (3.3) also reproduces the result in ref. [36] where the Schrödinger formalism to obtain vacuum averages for massive scalar fields near a reflecting wall has been used.

The mixed fluctuation in eq. (3.1) is given by

$$
\left\langle\phi^{2}\right\rangle_{\text {mixed }}^{(N)}=-\frac{1}{\pi}\left(\frac{M T}{2 \pi}\right)^{\frac{N-2}{2}} \sum_{n=1}^{\infty}\left[(2 T x)^{2}+n^{2}\right]^{\frac{2-N}{4}} K_{\frac{N-2}{2}}\left(\frac{M}{T} \sqrt{(2 T x)^{2}+n^{2}}\right) .
$$

It is a kind of non uniform "thermal" contribution that satisfies

$$
\left\langle\phi^{2}\right\rangle_{\text {mixed }}^{(N)}=-\left\langle\phi^{2}\right\rangle_{\text {thermal }}^{(N)}, \quad x=0 .
$$

Since $\left\langle\phi^{2}\right\rangle$ and $\left\langle T^{\mu \nu}\right\rangle$ share many common features, it is pedagogical to study $\left\langle\phi^{2}\right\rangle$ more closely. Unlike the vacuum fluctuation in eq. (3.3) that diverges as $x^{2-N}$ (see eq. (2.3)) when the wall is approached, the mixed contribution is free from boundary divergences (cf. eq. (3.5)). (Note that the thermal contribution is also divergence free since it is uniform.) 
As mentioned earlier, if one is interested in obtaining global quantities by integranting local quantities over space, then "cut-off procedures" are required to regulate integrations. It should be pointed out however that, whichever the "cut-off procedure" used, expressions for vacuum expectation values, such as that in eq. (3.3), are reliable as long as one is not too close to the reflecting wall.

By moving deep in the bulk of the cavity (i.e., $x \rightarrow \infty$ ), clearly the only contribution left behind in eq. (3.1) is the blackbody contribution $\left\langle\phi^{2}\right\rangle_{\text {thermal }}^{(N)}$. On the other hand, by progressively approaching the wall $(x \rightarrow 0)$ only the (divergent) $\left\langle\phi^{2}\right\rangle_{\text {vacuum }}^{(N)}$ is left at the end (see eqs. (3.1) and (3.5)). It should be stressed that the effects resulting of moving deep in the bulk and near the wall are equivalent, respectively, to those obtaining by keeping $x$ fixed raising the temperature $(T \rightarrow \infty)$ and lowering it $(T \rightarrow 0)$. Note that all these features are consistent with the asymptotic behaviour of the renormalized propagator (see eq. (2.6) and text just following it).

In order to find the leading correction to $\left\langle\phi^{2}\right\rangle_{\text {vacuum }}^{(N)}$ when $T x \ll 1$ (low temperature or near the wall), the expression for $\left\langle\phi^{2}\right\rangle_{\text {mixed }}^{(N)}$ in eq. (3.4) is expanded in powers of $T x$ whose leading term is that in eq. (3.5). By considering derivatives of $K_{\nu}(\mathrm{z})$, identities relating these functions [37], and omitting terms of higher powers, eq. (3.1) leads to

$$
\left\langle\phi^{2}\right\rangle^{(N)}=\left\langle\phi^{2}\right\rangle_{\text {vacuum }}^{(N)}+4 \pi x^{2}\left\langle\phi^{2}\right\rangle_{\text {thermal }}^{(N+2)}, \quad T x \ll 1 .
$$

Thus, at low temperature or near the wall, the leading correction to the vacuum fluctuation in $N$ dimensions is determined from the thermal fluctuation in $N+2$ dimensions (see eq. (3.2)).

The leading correction to $\left\langle\phi^{2}\right\rangle_{\text {thermal }}^{(N)}$ when $T x \gg 1$ (high temperature or deep in the bulk) can be obtained by replacing the sum in eq. (3.4) by an integration. The latter can be evaluated [37], resulting in

$$
\left\langle\phi^{2}\right\rangle^{(N)}=\left\langle\phi^{2}\right\rangle_{\text {thermal }}^{(N)}+\left\langle\phi^{2}\right\rangle_{\text {class }}^{(N)}, \quad T x \gg 1,
$$

where after reintroducing dimensionful $\hbar$ and $c$,

$$
\left\langle\phi^{2}\right\rangle_{\text {class }}^{(N)}:=\left\langle\phi^{2}\right\rangle_{\text {vacuum }}^{(N-1)} \frac{T}{\hbar c} .
$$

It should be pointed out that eq. (3.7) is exact up to exponentially small corrections which vanish as $T x \rightarrow \infty$ (note that $\left\langle\phi^{2}\right\rangle_{\text {vacuum }}^{(N)}$ in eq. (3.1) is cancelled by the subleading contribution in eq. (3.4)), and that it holds also for $N=2$ by considering $N=1$ in eq. (3.3). The behaviour corresponding to eqs. (3.7) and (3.8) is the first example in this paper of a local version of dimensional reduction. As will be seen in the next section, such a dimensional reduction will also be present in the behaviour of $\left\langle T^{\mu \nu}\right\rangle$.

As the dimensionful factor $1 / \hbar c$ appears in eq. (3.8), accordingly eq. (3.3) must be multiplied by $\hbar^{2-N / 2} c^{N / 2}$ and the argument of the corresponding Bessel function in eq. (3.3) must be multiplied by $c / \hbar$. Taking into account these amendments, it is easy to verify from eq. (3.8) that in general the correction $\left\langle\phi^{2}\right\rangle_{\text {class }}^{(N)}$ depends on $\hbar$, and will be "classical" only if the field is massless: if $M \rightarrow 0$, because of eq. (2.3), massless $\left\langle\phi^{2}\right\rangle_{\text {vacuum }}^{(N)}$ will depend on $\hbar$ 
and $c$ only through an overall factor $\hbar c$ which will cancel that in eq. (3.8). To illustrate this fact one sets $N=4$ in eq. (3.8) noting that $K_{1 / 2}(\mathrm{z})=\sqrt{(\pi / 2 \mathrm{z})} \exp (-\mathrm{z})$. It follows then

$$
\left\langle\phi^{2}\right\rangle_{\text {class }}^{(N=4)}=-\frac{T}{8 \pi x} \exp \left(-\frac{2 M c x}{\hbar}\right),
$$

which will clearly be classical only if $M=0$. At this point it should be remarked that in the study of the high temperature behaviour of massless fields, "classical" corrections linear in the temperature to blackbody expressions are a long known feature [6, 25, 26, 31]. The result above suggests that the designation "classical" for such corrections is not appropriate when the field has non-vanishing mass.

\section{Stress-energy-momentum tensor}

The local content of energy and momentum, as well as the stresses, of the hot scalar radiation in the infinity cavity are given by the ensemble average $\left\langle T^{\mu \nu}\right\rangle$, which can be formally obtained by acting with the differential operator

$$
\mathcal{D}^{\mu \nu}\left(\mathrm{x}, \mathrm{x}^{\prime}\right):=(1-2 \xi) \partial^{\mu} \partial^{\nu^{\prime}}-2 \xi \partial^{\mu} \partial^{\nu}+(2 \xi-1 / 2) \eta^{\mu \nu} \partial^{\lambda} \partial_{\lambda^{\prime}}+2 M^{2} \eta^{\mu \nu}(1 / 4-\xi)
$$

on the renormalized propagator in eq. (2.5),

$$
\begin{aligned}
\left\langle T^{\mu \nu}\right\rangle & =i \lim _{\mathrm{x}^{\prime} \rightarrow \mathrm{x}} \mathcal{D}^{\mu \nu}\left(\mathrm{x}, \mathrm{x}^{\prime}\right) G\left(\mathrm{x}, \mathrm{x}^{\prime}\right) \\
& =\left\langle T^{\mu \nu}\right\rangle_{\text {vacuum }}+\left\langle T^{\mu \nu}\right\rangle_{\text {mixed }}+\left\langle T^{\mu \nu}\right\rangle_{\text {thermal }},
\end{aligned}
$$

resulting,

$$
\left\langle T^{\mu \nu}\right\rangle^{(N)}=\operatorname{diag}\left(\rho^{(N)}, P_{\perp}^{(N)}, P_{\|}^{(N)}, \cdots, P_{\|}^{(N)}\right),
$$

where the terms in eq. (4.2) are all diagonal and each one of the elements in eq. (4.3) has the form in eq. (1.7). Accordingly, the energy density $\rho^{(N)}$ is given by

$$
\rho^{(N)}=\rho_{\text {vacuum }}^{(N)}+\rho_{\text {mixed }}^{(N)}+\rho_{\text {thermal }}^{(N)},
$$

with the blackbody energy density $\rho_{\text {thermal }}^{(N)}$ and the isotropic blackbody radiation pressure,

$$
P_{\perp \text { thermal }}^{(N)} \equiv P_{\| \text {thermal }}^{(N)}=2 \pi\left\langle\phi^{2}\right\rangle_{\text {thermal }}^{(N+2)},
$$

related by the equation of state

$$
\rho_{\text {thermal }}^{(N)}=(N-1) P_{\perp \text { thermal }}^{(N)}+M^{2}\left\langle\phi^{2}\right\rangle_{\text {thermal }}^{(N)} .
$$

Observing eq. (2.3), by setting $N=4$ and $M \rightarrow 0$ in eqs. (4.5) and (4.6) one reproduces eq. (1.2) as it should. Note that eq. (4.5) relates blackbody radiation pressure in $N$ dimensions with thermal fluctuation in $N+2$ dimensions (see eq. (3.2)).

The vacuum energy density in eq. (4.4) can be written in terms of vacuum fluctuations in eq. (3.3),

$$
\rho_{\text {vacuum }}^{(N)}=8 \pi(1-N)\left(\xi-\xi_{N}\right)\left\langle\phi^{2}\right\rangle_{\text {vacuum }}^{(N+2)}+M^{2}(1-4 \xi)\left\langle\phi^{2}\right\rangle_{\text {vacuum }}^{(N)},
$$


with $\left(\xi=\xi_{N}\right.$ is the conformal coupling)

$$
\xi_{N}:=\frac{N-2}{4(N-1)},
$$

and it satisfies the following equation of state,

$$
P_{\| \text {vacuum }}^{(N)}=-\rho_{\text {vacuum }}^{(N)},
$$

agreeing again with early calculations $[36,38]$ (it should be reported that an overall -1 factor is missing in eq. (B.11) of ref. [36]). Note that, at the wall, the vacuum energy density diverges as $x^{-N}$ if $\xi \neq \xi_{N}$, and as $x^{2-N}$ if $\xi=\xi_{N}$ with $M \neq 0$.

To complete the expression for the energy density in eq. (4.4), the mixed contribution is (see also eq. (B.1))

$$
\begin{aligned}
\rho_{\text {mixed }}^{(N)}= & 2\left(\frac{M T}{2 \pi}\right)^{N / 2} \sum_{n=1}^{\infty}\left[(2 T x)^{2}+n^{2}\right]^{-(N+4) / 4} \\
& \times\left\{4\left[(N-1)\left(\xi-\xi_{N}\right)\left(4 T^{2} x^{2}+n^{2}\right)-\xi N n^{2}\right] K_{\frac{N}{2}}\left(\frac{M}{T} \sqrt{(2 T x)^{2}+n^{2}}\right)\right. \\
& \left.+\frac{M}{T}\left[(4 T x)^{2}(\xi-1 / 4)-n^{2}\right] \sqrt{(2 T x)^{2}+n^{2}} K_{\frac{N-2}{2}}\left(\frac{M}{T} \sqrt{(2 T x)^{2}+n^{2}}\right)\right\} .
\end{aligned}
$$

As happens to all the mixed contributions in this paper, $\rho_{\text {mixed }}^{(N)}$ is finite at the wall $(x=0)$. In particular, corresponding to eq. (3.5), it satisfies

$$
\rho_{\text {mixed }}^{(N)}=-\rho_{\text {thermal }}^{(N)}, \quad \xi=1 / 4, \quad x=0 .
$$

The vacuum contribution and the mixed contribution in $P_{\perp}^{(N)}$ vanish identically, resulting that the pressure on the wall is the only component in eq. (4.3) which is uniform, i.e.,

$$
P_{\perp}^{(N)}=P_{\perp \text { thermal }}^{(N)},
$$

where the blackbody pressure in eq. (4.12) is given as in eq. (4.5). Finally,

$$
P_{\|}^{(N)}=P_{\| \text {vacuum }}^{(N)}+P_{\| \text {mixed }}^{(N)}+P_{\| \text {thermal }}^{(N)},
$$

where the thermal and vacuum contributions are given by eqs. (4.5) and (4.9). The mixed contribution is given by (see also eq. (B.2))

$$
\begin{aligned}
P_{\| \text {mixed }}^{(N)}= & -2\left(\frac{M T}{2 \pi}\right)^{N / 2} \sum_{n=1}^{\infty}\left[(2 T x)^{2}+n^{2}\right]^{-(N+4) / 4} \\
\times & \left\{4\left[(N-1)\left(\xi-\xi_{N}\right)\left(4 T^{2} x^{2}+n^{2}\right)-(\xi-1 / 4) N n^{2}\right] K_{\frac{N}{2}}\left(\frac{M}{T} \sqrt{(2 T x)^{2}+n^{2}}\right)\right. \\
& \left.+16 M T x^{2}(\xi-1 / 4) \sqrt{(2 T x)^{2}+n^{2}} K_{\frac{N-2}{2}}\left(\frac{M}{T} \sqrt{(2 T x)^{2}+n^{2}}\right)\right\}
\end{aligned}
$$

Corresponding to eqs. (3.5) and (4.11),

$$
P_{\| \text {mixed }}^{(N)}=-P_{\| \text {thermal }}^{(N)}, \quad \xi=1 / 4, \quad x=0 .
$$


One can check the consistency of the formulas just obtained for the components of $\left\langle T^{\mu \nu}\right\rangle$ by calculating its trace $\left\langle T_{\mu}^{\mu}\right\rangle$. Since the background is flat the stress-energymomentum tensor is expected to be traceless when $\xi=\xi_{N}$ and $M=0$. Indeed, a straightforward calculation yields

$$
\left\langle T^{\mu}{ }_{\mu}\right\rangle_{\text {thermal }}^{(N)}=M^{2}\left\langle\phi^{2}\right\rangle_{\text {thermal }}^{(N)},
$$

and

$$
\left\langle T_{\mu}^{\mu}\right\rangle_{\text {vacuum }}^{(N)}=M^{2}\left\langle\phi^{2}\right\rangle_{\text {vacuum }}^{(N)}, \quad\left\langle T_{\mu}^{\mu}\right\rangle_{\text {mixed }}^{(N)}=M^{2}\left\langle\phi^{2}\right\rangle_{\text {mixed }}^{(N)}, \quad \xi=\xi_{N} .
$$

Using now eq. (4.2), one gets

$$
\left\langle T_{\mu}^{\mu}\right\rangle^{(N)}=M^{2}\left\langle\phi^{2}\right\rangle^{(N)}, \quad \xi=\xi_{N},
$$

with the mean square field fluctuation given in eq. (3.1).

The asymptotic behaviour at low temperature (or near the wall) of $\rho^{(N)}$ and $P_{\|}^{(N)}$ in eq. (4.3) can be obtained by simply setting $T x=0$ in the expressions for their mixed contributions. After some algebra it results,

$$
P_{\|}^{(N)}=-\rho^{(N)}=-\rho_{\text {vacuum }}^{(N)}-(1-4 \xi) P_{\perp \text { thermal }}^{(N)}, \quad T x \ll 1,
$$

up to zeroth order in $T x$. It should be pointed out that, unlike eq. (3.6), the correction to the vacuum contribution in eq. (4.19) does not depend on $x$. This can be understood by recalling that $\left\langle T^{\mu \nu}\right\rangle$ is obtained from a propagator by applying to the latter a differential operator (see eq. (4.2)). Regarding $P_{\perp}^{(N)}$, eq. (4.12) holds everywhere and at all temperatures.

The asymptotic behaviour of the energy density $\rho^{(N)}$ in eq. (4.3) at high temperature (or deep in the bulk) can be found by proceeding as in deriving eqs. (3.7) and (3.8). Namely, noting eq. (4.4), the sums in the expression for the mixed contribution in eq. (B.1) are replaced by integrations that can be solved [37]. Up to exponentially small corrections one finds,

$$
\rho^{(N)}=\rho_{\text {thermal }}^{(N)}+\rho_{\text {class }}^{(N)}, \quad T x \gg 1,
$$

where

$$
\rho_{\text {class }}^{(N)}=(1-4 \xi)\left[2 \pi(N-2)\left\langle\phi^{2}\right\rangle_{\text {vacuum }}^{(N+1)}+M^{2}\left\langle\phi^{2}\right\rangle_{\text {vacuum }}^{(N-1)}\right] T,
$$

holding also for $N=2$. Observing now the expression for the vacuum energy density in eq. (4.7), it follows from eq. (4.21) when $N>2$,

$$
\left(\xi-\xi_{N-1}\right) \rho_{\text {class }}^{(N)}=(\xi-1 / 4)\left[\rho_{\text {vacuum }}^{(N-1)}-\frac{M^{2} c^{2}}{(N-2) \hbar^{2}}\left\langle\phi^{2}\right\rangle_{\text {vacuum }}^{(N-1)}\right] \frac{T}{\hbar c} .
$$

Note that $\xi_{N-1}$ in eq. (4.22) is the conformal coupling in $N-1$ spacetime dimensions (cf. eq. (4.8)), and that dimensionful $\hbar$ and $c$ were reintroduced (as in eq. (3.8)) to study the "classical" nature of $\rho_{\text {class }}^{(N)}$ shortly. The statement in eq. (4.22) is another instance in the paper of a local dimensional reduction, i.e., corrections to $N$-dimensional blackbody expressions are obtained from $(N-1)$-dimensional vacuum expectation values of local quantities. 
Turning now to the nature of $\rho_{\text {class }}^{(N)}$ in eq. (4.22), by considering eq. (4.7) and that when $M=0$ the vacuum fluctuation $\left\langle\phi^{2}\right\rangle_{\text {vacuum }}^{(N)}$ depends on $\hbar$ and $c$ only through the overall factor $\hbar c$ (see discussion in the paragraph containing eq. (3.9)), it follows that $\rho_{\text {class }}^{(N)}$ will be classical only if the field is massless: by setting $M \rightarrow 0$ in eq. (4.22), the overall factor $\hbar c$ that arises in $\rho_{\text {vacuum }}^{(N-1)}$ will cancel that in $T / \hbar c$.

The same line of reasoning applied to eqs. (4.13) and (B.2) yields, up to exponentially small corrections,

$$
P_{\|}^{(N)}=P_{\| \text {thermal }}^{(N)}+P_{\| \text {class }}^{(N)}, \quad T x \gg 1,
$$

where,

$$
P_{\| \text {class }}^{(N)}=P_{\| \text {vacuum }}^{(N-1)} \frac{T}{\hbar c}
$$

(see eqs. (4.5) and (4.9)). Again, local dimensional reduction operates and $P_{\| \text {class }}^{(N)}$ is classical only if the field is massless.

Noticing eqs. (3.8), (4.9), (4.22) and (4.24), it follows the equation of state,

$$
\left(\xi_{N-1}-\xi\right) \rho_{\text {class }}^{(N)}=(\xi-1 / 4)\left[P_{\| \text {class }}^{(N)}+\frac{M^{2}}{N-2}\left\langle\phi^{2}\right\rangle_{\text {class }}^{(N)}\right]
$$

relating corrections to the blackbody contributions. If one is interested in $N=2$, eq. (4.25) must be multiplied by $N-2$ before setting $N=2$ (cf. eq. (4.21)).

Before ending this section it should be remarked that, because of eq. (4.12),

$$
P_{\perp \text { class }}^{(N)}=0,
$$

which is consistent with dimensional reduction since $P_{\perp \text { vacuum }}^{(N)}=0$ for arbitrary $N$. If the wall parallel to that at $x=0$ were brought from infinity to a finite distance, the zero in eq. (4.26) should give place to the well known thermal Casimir pressure [4, 31, 39].

\section{Ultralight scalar radiation}

When $T \gg M$, the leading order expressions for $\left\langle\phi^{2}\right\rangle^{(N)}$ and $\left\langle T^{\mu \nu}\right\rangle^{(N)}$ in eqs. (3.1) and (4.3) can be obtained simply by taking $M \rightarrow 0$ and considering eq. (2.3). For completeness, the relevant quantities are given below. They are the masless expressions,

$$
\begin{aligned}
\left\langle\phi^{2}\right\rangle_{\text {vacuum }}^{(N)} & =-\frac{1}{\left(2 \pi^{1 / 2}\right)^{N}} \Gamma\left(\frac{N-2}{2}\right) x^{2-N}, \\
\left\langle\phi^{2}\right\rangle_{\text {mixed }}^{(N)} & =-\frac{1}{2 \pi^{N / 2}} \Gamma\left(\frac{N-2}{2}\right) T^{N-2} \sum_{n=1}^{\infty}\left[(2 T x)^{2}+n^{2}\right]^{(2-N) / 2}, \\
\left\langle\phi^{2}\right\rangle_{\text {thermal }}^{(N)} & =\frac{1}{2 \pi^{N / 2}} \Gamma\left(\frac{N-2}{2}\right) \zeta(N-2) T^{N-2},
\end{aligned}
$$

and

$$
\begin{aligned}
\rho_{\text {mixed }}^{(N)}= & \frac{4}{\pi^{N / 2}} \Gamma\left(\frac{N}{2}\right) T^{N} \sum_{n=1}^{\infty}\left[(2 T x)^{2}+n^{2}\right]^{-(N+2) / 2} \\
& \times\left[(N-1)\left(\xi-\xi_{N}\right)\left(4 T^{2} x^{2}+n^{2}\right)-\xi N n^{2}\right],
\end{aligned}
$$




$$
\begin{aligned}
P_{\| \text {mixed }}^{(N)}= & -\frac{4}{\pi^{N / 2}} \Gamma\left(\frac{N}{2}\right) T^{N} \sum_{n=1}^{\infty}\left[(2 T x)^{2}+n^{2}\right]^{-(N+2) / 2} \\
& \times\left[(N-1)\left(\xi-\xi_{N}\right)\left(4 T^{2} x^{2}+n^{2}\right)-(\xi-1 / 4) N n^{2}\right] .
\end{aligned}
$$

Any component of massless $\left\langle T^{\mu \nu}\right\rangle^{(N)}$ (cf. ref. [34]) can be obtained by using eqs. (5.1) and (5.2) in the general expressions found in the previous section. It is worth remarking that, noticing $\left\langle\phi^{2}\right\rangle^{(N)}$ given by eq. (3.1) and the functional dependence on $N$ in eqs. (5.1), one must have $N>3$ in order to avoid divergences in the mean square field fluctuation of hot massless scalar radiation (if $T=0$, one must have $N>2$ ).

\section{Neumann's boundary condition}

Another boundary condition also used to prevent fluxes through the plane wall is Neumann's. Instead of taking $\phi$ itself to vanish at the wall, one takes,

$$
\frac{\partial \phi}{\partial x}=0, \quad x=0 .
$$

Accordingly, the new Feynman propagator $G_{\mathcal{F}}\left(\mathrm{x}, \mathrm{x}^{\prime}\right)$ at finite temperature $T$ is obtained by solving again eq. (2.1), but now observing eq. (6.1) (see appendix A). The corresponding expression for $G_{\mathcal{F}}\left(\mathrm{x}, \mathrm{x}^{\prime}\right)$ can be obtained from that in eq. (2.2) by replacing the minus sign between the terms containing the modified Bessel functions by a plus sign. It follows then that eq. (2.4) holds with $G_{0}\left(\mathrm{x}, \mathrm{x}^{\prime}\right)$ and $G_{\text {thermal }}\left(\mathrm{x}, \mathrm{x}^{\prime}\right)$ still denoting the vacuum and the thermal propagators in Minkowski spacetime, respectively, whereas $G_{\text {vacuum }}\left(\mathrm{x}, \mathrm{x}^{\prime}\right)$ and $G_{\text {mixed }}\left(\mathrm{x}, \mathrm{x}^{\prime}\right)$ are the negative of the Dirichlet ones. Noting these modifications the renormalized propagator is given as in eq. (2.5). Consequently the ensemble average of a quantity $\mathcal{A}$ (cf. eq. (1.7)) near the Neumann wall can be obtained as follows,

$$
\left\langle\mathcal{A}_{\mathcal{N}}\right\rangle=-\left\langle\mathcal{A}_{\mathcal{D}}\right\rangle_{\text {vacuum }}-\left\langle\mathcal{A}_{\mathcal{D}}\right\rangle_{\text {mixed }}+\langle\mathcal{A}\rangle_{\text {thermal }}
$$

where $\left\langle\mathcal{A}_{\mathcal{D}}\right\rangle_{\text {vacuum }}$ and $\left\langle\mathcal{A}_{\mathcal{D}}\right\rangle_{\text {mixed }}$ are expressions for Dirichlet's wall. It is worth remarking that eq. (6.2) generalizes to finite temperature $T$ the well known identity,

$$
\left\langle\mathcal{A}_{\mathcal{N}}\right\rangle_{\text {vacuum }}=-\left\langle\mathcal{A}_{\mathcal{D}}\right\rangle_{\text {vacuum }}
$$

As $T \rightarrow 0$, eq. (6.2) leads to eq. (6.3). Clearly

$$
\left\langle\mathcal{A}_{\mathcal{N}}\right\rangle_{\text {mixed }}=-\left\langle\mathcal{A}_{\mathcal{D}}\right\rangle_{\text {mixed }}
$$

also holds.

Considering eq. (6.2) and the corresponding Dirichlet expressions computed in the previous sections, one can determine the asymptotic thermal behaviours of $\left\langle\phi^{2}\right\rangle$ and $\left\langle T^{\mu \nu}\right\rangle$ for the Neumann boundary condition at the plane wall. In order to simplify the notation and to allow ready comparison with the Dirichlet boundary condition, in the rest of the section expressions denoted as $\langle\mathcal{A}\rangle,\langle\mathcal{A}\rangle_{\text {vacuum }}$ and $\langle\mathcal{A}\rangle_{\text {mixed }}$ correspond to those for Neumann's boundary condition (cf. eqs. (6.3) and (6.4)). 
Begining with the mean square field fluctuation, now $\left\langle\phi^{2}\right\rangle_{\text {mixed }}^{(N)}=\left\langle\phi^{2}\right\rangle_{\text {thermal }}^{(N)}$ at $x=0$, replacing eq. (3.5) and leading to

$$
\left\langle\phi^{2}\right\rangle^{(N)}=\left\langle\phi^{2}\right\rangle_{\text {vacuum }}^{(N)}+2\left\langle\phi^{2}\right\rangle_{\text {thermal }}^{(N)}, \quad T x \ll 1 .
$$

Thus eq. (6.5) shows that, unlike the Dirichlet $\left\langle\phi^{2}\right\rangle$ (cf. eq. (3.6)), the thermal behaviour of $\left\langle\phi^{2}\right\rangle$ near the Neumann wall is (in leading order) uniform, namely, twice that of a blackbody. Perhaps it should be noted that Neumann vacuum fluctuations are plagued by the same boundary divergences as their Dirichlet counterparts (more precisely, they differ by a minus sign).

When $T x \gg 1$ (high temperature or deep in the bulk), because of eqs. (6.3) and (6.4) one can see that the local version of dimensional reduction also operates in the bulk of a "Neumann cavity". Regarding $\left\langle\phi^{2}\right\rangle$, eqs. (3.7) and (3.8) still apply, with the vacuum expectation value in eq. (3.8) corresponding now to Neumann's boundary condition (cf. eq. (6.3)).

The ensemble average of the stress-energy-momentum tensor is given again by the sum of diagonal terms as in eq. (4.2), resulting in eq. (4.3) now adapted for Neumann's boundary condition at the plane wall (see eqs. (6.2), (6.3) and (6.4)). Clearly eqs. (4.7), (4.9) and (4.12) remain unchanged, whereas eqs. (4.11) and (4.15) are replaced by (for $\xi=1 / 4$ and $x=0) \rho_{\text {mixed }}^{(N)}=\rho_{\text {thermal }}^{(N)}$ and $P_{\| \text {mixed }}^{(N)}=P_{\| \text {thermal }}^{(N)}$. Regarding traces, one sees that eqs. (4.17) and (4.18) still hold.

At low temperature (or near the Neumann wall), $\rho^{(N)}$ and $P_{\|}^{(N)}$ are given by,

$$
\rho^{(N)}=\rho_{\text {vacuum }}^{(N)}+(2 N-3+4 \xi) P_{\perp \text { thermal }}^{(N)}+2 M^{2}\left\langle\phi^{2}\right\rangle_{\text {thermal }}^{(N)}, \quad T x \ll 1,
$$

and

$$
P_{\|}^{(N)}=-\rho_{\text {vacuum }}^{(N)}+(3-4 \xi) P_{\perp \text { thermal }}^{(N)}, \quad T x \ll 1,
$$

up to zeroth order in $T x$. These equations replace eq. (4.19). It should be noted in particular that the first equality in eq. (4.19) no longer holds near the Newmann wall. As mentioned earlier in this section "local dimensional reduction" also operates for the Neumann boundary condition, i.e., eqs. (4.20) and (4.22) hold with the vacuum expectation values corresponding to Neumann's vacuum (cf. eqs. (6.3)). Perhaps this is a good place to remark that by setting $N=4$ and $M=0$, the expressions in this section reproduce consistently the results in refs. $[2,3]$.

It is worth mentioning that a curiosity spotted in ref. [2] is associated to the fact that the "local dimensional reduction" operates for both boundary conditions at the plane wall. Quoted from ref. [2]: “... the correction to the Planckian energy density at high temperature is for minimal coupling, ... , exactly three times that for conformal coupling, irrespective of the boundary conditions ... ". Noting that eq. (4.21) applies equally to both boundary conditions, it follows that,

$$
\rho_{\text {class }}^{(N)}(\xi=0)=(N-1) \rho_{\text {class }}^{(N)}\left(\xi=\xi_{N}\right) .
$$

Now one sees that the curiosity spotted in ref. [2] is the content of eq. (6.7) for $N=4$.

Comparison of $d \rho^{(N)} / d T$ for the Dirichlet and Neumann boundary conditions (denoted below by $c_{\mathcal{D}}$ and $c_{\mathcal{N}}$, respectively) reveals contrasting features. For simplicity a massless 
scalar field will be considered (i.e., $M=0$ ). Noting eqs. (4.19), (6.6) and (4.6), it follows that,

$$
c_{\mathcal{D}}=\frac{1-4 \xi}{N-1} c_{V}, \quad c_{\mathcal{N}}=\frac{2 N-3+4 \xi}{N-1} c_{V}, \quad T x \ll 1,
$$

where $c_{V}:=d \rho_{\text {thermal }}^{(N)} / d T$ is the familiar (nonnegative) blackbody specific heat per unit of volume. By examining eq. (6.8) one sees that when $\xi>1 / 4, c_{\mathcal{D}}<0$ and $c_{\mathcal{N}}>0$. The physical interpretation of these inequalities is as follows. When $\xi>1 / 4$, for increasing temperature $T$, the energy density near the Dirichlet wall decreases, whereas near the Neumann wall it increases. Deep in the bulk, both $c_{\mathcal{D}}$ and $c_{\mathcal{N}}$ behave in leading order as $c_{V}$ (cf. eq. (4.20)), i.e., the energy density increases as the temperature increases.

\section{Conclusion}

Blackbody radiation of a massive scalar field is a topic of interest both in cosmology and high energy physics. This work examined the local behaviour (in contrast to the usual global approach) of hot scalar radiation near infinite plane Dirichlet and Neumann walls in flat spacetime with $N$ dimensions. The Dirichlet boundary condition (which is conformal invariant) and the Neumann boundary condition (which is not conformal invariant) aimed to model the real reflecting wall of a large cavity. The scalar field $\phi$ was taken to be neutral, with mass $M$, and arbitrarily coupled to the absent curvature (arbitrary $\xi$ ). Using the "point-splitting" procedure and the Schwinger "proper time" representation of the Feynman propagator at finite temperature $T$, new formulas for $\left\langle\phi^{2}\right\rangle$ and $\left\langle T^{\mu \nu}\right\rangle$ were derived, and those for $\left\langle T^{\mu \nu}\right\rangle$ were shown to reproduce known results when $N=4, M=0$, and $\xi=0$ or $1 / 6$.

The ensemble averages $\left\langle\phi^{2}\right\rangle$ and $\left\langle T^{\mu \nu}\right\rangle$ were expressed as in eq. (1.7) where the vacuum contributions revealed divergences at the wall that are typical of the use of idealized boundary conditions. It was mentioned that divergences of these kind do not affect the other contributions in eq. (1.7), both depending on $T$ and vanishing when $T=0$. It is worth recalling that if the distribution in eq. (1.1) were used to compute averages, only the thermal contribution (the blackbody contribution) would appear in eq. (1.7). The term denoted by "mixed" in eq. (1.7) has hybrid nature, and connects the vacuum and the blackbody contributions. Such a hybrid nature is the ingredient responsible for contrasts in the thermal behaviour corresponding to the Dirichlet and Neumann boundary conditions.

The asymptotic behaviours of $\left\langle\phi^{2}\right\rangle$ and $\left\langle T^{\mu \nu}\right\rangle$ at low $(T x \ll 1)$ and high $(T x \gg 1)$ temperature were thoroughly studied. At low temperature, the dependence on $T$ was shown to be given by the leading correction to the Dirichlet or Neumann vacuum contributions. Regarding the regime of high temperature, it was shown that corrections to the blackbody contributions carry Planck's constant if $M \neq 0$ (i.e., they are not "classical", in general). Another feature spotted is a "local dimensional reduction" by means of which one can say the corrections to blackbody contributions by looking at vacuum contributions in one less dimension. At this point it should be stressed that the "local dimensional reduction" is present for massless and massive fields, and for both boundary conditions considered, suggesting that it may be a common feature of more general setups. 
It is worth remarking that although nowhere in the text any restriction on the values of the curvature coupling parameter $\xi$ was imposed, under certain plausible assumptions thermodynamic arguments in ref. [34] suggest that not all values of $\xi$ are consistent with stable thermodynamic equilibrium.

An extension of this work would be to look at the generality of the "local dimensional reduction" when the background is not strictly flat (especially when an event horizon is present). Another pertinent extension would be to study how the "local dimensional reduction" operates when the plane wall is replaced by a spherical shell. The study of a charged scalar, as well as of fields of higher spins, may also reveal new interesting effects.

\section{A Finite temperature propagator}

The geometry of the $N$-dimensional spacetime is given by

$$
d s^{2}=d t^{2}-d x^{2}-d y^{2}-d z^{2}-\cdots
$$

The coordinate $x_{1}:=i t$ is taken to be real with period $\beta:=1 / T$. By convenience $x_{0}:=i x$ is also analytically continued to real values. Considering further $x_{2}:=y$ and $x_{3}:=z$, eq. (A.1) becomes,

$$
d s^{2}=d x_{0}^{2}-d x_{1}^{2}-d x_{2}^{2}-d x_{3}^{2}-\cdots-d x_{N-1}^{2},
$$

which with the boundary condition,

$$
\psi\left(x_{0}, x_{1}, x_{2}, \cdots, x_{N-1}\right)=\psi\left(x_{0}, x_{1}+\beta, x_{2}, \cdots, x_{N-1}\right)
$$

characterizes a cylindrical spacetime. The Dirichlet boundary condition at $x=0$ is implemented by taking,

$$
\psi\left(x_{0}=0, x_{1}, x_{2}, \cdots, x_{N-1}\right)=0 .
$$

Since $\square_{\mathrm{x}}:=\partial_{0}^{2}-\partial_{1}^{2}-\partial_{2}^{2}-\partial_{3}^{2}-\cdots-\partial_{N-1}^{2}$, the eigenfunctions of the operator $\square_{\mathrm{x}}+M^{2}$ are given by

$$
\psi_{\omega, \mathbf{k}}(\mathrm{x})=\eta \sin \left(\omega x_{0}\right) \exp (i \mathbf{k} \cdot \mathbf{x})
$$

$\mathbf{x}:=\left(x_{1}, x_{2}, \cdots, x_{N-1}\right)$, and where $\eta, \omega$ as well as the components of $\mathbf{k}:=$ $\left(k_{1}, k_{2}, \cdots, k_{N-1}\right)$ are constants. The corresponding eigenvalues are

$$
E_{\omega, \mathbf{k}}=\mathbf{k} \cdot \mathbf{k}-\omega^{2}+M^{2},
$$

and due to eq. (A.3) $k_{1}=2 \pi n / \beta$ with $n$ an integer. The sine function in eq. (A.5) ensures that eq. (A.4) holds.

One can check now that the Feynman propagator is given by

$$
G_{\mathcal{F}}\left(\mathrm{x}, \mathrm{x}^{\prime}\right)=-i \sum_{n=-\infty}^{\infty} \int_{0}^{\infty} d \tau \int_{0}^{\infty} d \omega \int_{-\infty}^{\infty} d k_{2} \cdots \int_{-\infty}^{\infty} d k_{N-1} e^{-i \tau E_{\omega, \mathbf{k}}} \psi_{\omega, \mathbf{k}}(\mathrm{x}) \psi_{\omega, \mathbf{k}}^{*}\left(\mathrm{x}^{\prime}\right),
$$


simply by applying $\square_{\mathrm{x}}+M^{2}$ to eq. (A.7),

$$
\begin{aligned}
\left(\square_{\mathrm{x}}+M^{2}\right) G_{\mathcal{F}}\left(\mathrm{x}, \mathrm{x}^{\prime}\right)= & \sum_{n=-\infty}^{\infty} \int_{0}^{\infty} d \omega \int_{-\infty}^{\infty} d k_{2} \cdots \int_{-\infty}^{\infty} d k_{N-1} \psi_{\omega, \mathbf{k}}(\mathrm{x}) \psi_{\omega, \mathbf{k}}^{*}\left(\mathrm{x}^{\prime}\right) \\
& \times \int_{0}^{\infty} d \tau \frac{d}{d \tau} e^{-i \tau E_{\omega, \mathbf{k}}}
\end{aligned}
$$

To make the integration over the "proper time" $\tau$ in eq. (A.8) convergent, $M^{2}$ in eq. (A.6) is taken to have an infinitesimal negative imaginary part, resulting that the integration over $\tau$ yields minus unity. By considering eq. (A.5) in eq. (A.8) with $\eta$ such that

$$
|\eta|^{2}=2^{3-N} \pi^{1-N} \beta^{-1},
$$

the integrations over the components of $\mathbf{k}$ give the usual representation of the $\delta$-function. Noting that $\mathrm{x}$ and $\mathrm{x}^{\prime}$ are at the same side of the wall, using Poisson's formula,

$$
\sum_{n=-\infty}^{\infty} \delta(\lambda-2 \pi n)=\frac{1}{2 \pi} \sum_{n=-\infty}^{\infty} e^{-i n \lambda}
$$

and that $[40]$

$$
\frac{2}{\pi} \int_{0}^{\infty} d \omega \sin (\omega x) \sin \left(\omega x^{\prime}\right)=\delta\left(x-x^{\prime}\right)-\delta\left(x+x^{\prime}\right)
$$

one sees that the right hand side of eq. (A.8) is indeed minus the delta function in the $N$-dimensional cylindrical spacetime (cf. eqs. (A.2) and (A.3)).

In order to arrive in eq. (2.2), eq. (A.5) is used in eq. (A.7) noticing eq. (A.9). In so doing a factor arises that can be conveniently manipulated as follows,

$$
\begin{aligned}
\sum_{n=-\infty}^{\infty} e^{-i \tau\left(4 \pi^{2} n^{2} / \beta^{2}\right)+i(2 \pi n / \beta)\left(x_{1}-x_{1}^{\prime}\right)} & =\sum_{n=-\infty}^{\infty} \int_{-\infty}^{\infty} d \lambda \delta(\lambda-2 \pi n) e^{-i \tau\left(\lambda^{2} / \beta^{2}\right)+i(\lambda / \beta)\left(x_{1}-x_{1}^{\prime}\right)} \\
& =\frac{1}{2 \pi} \sum_{n=-\infty}^{\infty} \int_{-\infty}^{\infty} d \lambda e^{-i \tau\left(\lambda^{2} / \beta^{2}\right)+i(\lambda / \beta)\left(x_{1}-x_{1}^{\prime}-n \beta\right)}
\end{aligned}
$$

where eq. (A.10) has been used in the last step. Now all integrations other than that over $\tau$ can be promptly evaluated, and the final integration over $\tau$ yields the modified Bessel functions [37]. The expression in eq. (2.2) is obtained by analytically continuing back to reall values of $t=-i x_{1}$ and $x=-i x_{0}$, and after going back to the original coordinates in eq. (A.1).

Instead of eq. (A.4), the Neumann boundary condition in eq. (6.1) is implemented by taking

$$
\frac{\partial \psi}{\partial x_{0}}=0, \quad x_{0}=0
$$

Accordingly, the eigenfunctions of the operator $\square_{\mathrm{x}}+M^{2}$ are now given by

$$
\psi_{\omega, \mathbf{k}}(\mathrm{x})=\eta \cos \left(\omega x_{0}\right) \exp (i \mathbf{k} \cdot \mathbf{x}),
$$

replacing eq. (A.5). Considering eq. (A.11), one follows the same line of reasoning as above, ending with eq. (2.2) but now with the minus sign into the square bracket giving place to a plus sign. 


\section{B Alternative expressions for certain averages}

The following formulas are alternative expressions to $\rho_{\text {mixed }}^{(N)}$ and $P_{\| \text {mixed }}^{(N)}$ given in eqs. (4.10) and (4.14),

$$
\begin{aligned}
\rho_{\text {mixed }}^{(N)}= & 2\left(\frac{M T}{2 \pi}\right)^{N / 2} \sum_{n=1}^{\infty}\left[(2 T x)^{2}+n^{2}\right]^{-N / 4}\left\{2(1-2 \xi) K_{\frac{N}{2}}\left(\frac{M}{T} \sqrt{(2 T x)^{2}+n^{2}}\right)\right. \\
& \left.+\frac{M}{T}\left[(4 T x)^{2}(\xi-1 / 4)-n^{2}\right]\left[(2 T x)^{2}+n^{2}\right]^{-1 / 2} K_{\frac{N+2}{2}}\left(\frac{M}{T} \sqrt{(2 T x)^{2}+n^{2}}\right)\right\},
\end{aligned}
$$

and

$$
\begin{aligned}
P_{\| \text {mixed }}^{(N)}= & -2\left(\frac{M T}{2 \pi}\right)^{N / 2} \sum_{n=1}^{\infty}\left[(2 T x)^{2}+n^{2}\right]^{-N / 4}\left\{2(1-2 \xi) K_{\frac{N}{2}}\left(\frac{M}{T} \sqrt{(2 T x)^{2}+n^{2}}\right)\right. \\
& \left.+16 M T x^{2}(\xi-1 / 4)\left[(2 T x)^{2}+n^{2}\right]^{-1 / 2} K_{\frac{N+2}{2}}\left(\frac{M}{T} \sqrt{(2 T x)^{2}+n^{2}}\right)\right\} .
\end{aligned}
$$

They lead to those in the text containing $K_{\nu}(z)$ of lower $\nu$ by considering known identities relating Bessel functions of different orders [37].

\section{Acknowledgments}

This work was partially supported by the Brazilian research agencies CAPES, CNPq and FAPEMIG.

Open Access. This article is distributed under the terms of the Creative Commons Attribution License (CC-BY 4.0), which permits any use, distribution and reproduction in any medium, provided the original author(s) and source are credited.

\section{References}

[1] F. Bezrukov and M. Shaposhnikov, The standard model Higgs boson as the inflaton, Phys. Lett. B 659 (2008) 703 [arXiv:0710.3755] [INSPIRE].

[2] G. Kennedy, R. Critchley and J.S. Dowker, Finite temperature field theory with boundaries: stress tensor and surface action renormalization, Annals Phys. 125 (1980) 346 [INSPIRE].

[3] S. Tadaki and S. Takagi, Casimir effect at finite temperature, Prog. Theor. Phys. 75 (1986) 262 [INSPIRE].

[4] K.A. Milton, The Casimir effect, physical manifestations of zero-point energy, World Scientific, U.S.A. (2001).

[5] M. Bordag, U. Mohideen and V.M. Mostepanenko, New developments in the Casimir effect, Phys. Rept. 353 (2001) 1 [quant-ph/0106045] [INSPIRE].

[6] R. Balian and B. Duplantier, Electromagnetic waves near perfect conductors. II. Casimir effect, Annals Phys. 112 (1978) 165 [InSPIRE].

[7] B.S. DeWitt, Quantum field theory in curved spacetime, Phys. Rept. 19 (1975) 295 [INSPIRE]. 
[8] D. Deutsch and P. Candelas, Boundary effects in quantum field theory, Phys. Rev. D 20 (1979) 3063 [inSPIRE].

[9] G. Barton and N. Dombey, Casimir effect for massive photons, Nature 311 (1984) 336 [INSPIRE].

[10] P.C.W. Davies and D.J. Toms, Boundary effects and the massless limit of the photon, Phys. Rev. D 31 (1985) 1363 [INSPIRE].

[11] N. Graham et al., The Dirichlet Casimir problem, Nucl. Phys. B 677 (2004) 379 [hep-th/0309130] [INSPIRE].

[12] S.A. Fulling, Vacuum energy density and pressure near boundaries, Int. J. Mod. Phys. A 25 (2010) 2364 [inSPIRE].

[13] K.A. Milton, Hard and soft walls, Phys. Rev. D 84 (2011) 065028 [arXiv:1107.4589] [INSPIRE].

[14] J.D. Bouas et al., Investigating the spectral geometry of a soft wall, Proc. Symp. Pure Math. 84 (2012) 139 [arXiv:1106.1162] [INSPIRE].

[15] F.D. Mazzitelli, J.P. Nery and A. Satz, Boundary divergences in vacuum self-energies and quantum field theory in curved spacetime, Phys. Rev. D 84 (2011) 125008 [arXiv:1110.3554] [INSPIRE].

[16] N. Bartolo and R. Passante, Electromagnetic-field fluctuations near a dielectric-vacuum boundary and surface divergences in the ideal conductor limit, Phys. Rev. A 86 (2012) 012122 [arXiv: 1204.6475].

[17] K.A. Milton, K.V. Shajesh, S.A. Fulling and P. Parashar, How does Casimir energy fall? IV. Gravitational interaction of regularized quantum vacuum energy, Phys. Rev. D 89 (2014) 064027 [arXiv: 1401.0784] [INSPIRE].

[18] G. Kennedy, Finite temperature field theory with boundaries: the photon field, Annals Phys. 138 (1982) 353 [INSPIRE].

[19] A. Romeo and A.A. Saharian, Casimir effect for scalar fields under Robin boundary conditions on plates, J. Phys. A 35 (2002) 1297 [hep-th/0007242] [INSPIRE].

[20] L.H. Ford and N.F. Svaiter, Vacuum energy density near fluctuating boundaries, Phys. Rev. D 58 (1998) 065007 [quant-ph/9804056] [INSPIRE].

[21] J.S. Dowker and G. Kennedy, Finite temperature and boundary effects in static space-times, J. Phys. A 11 (1978) 895 [inSPIRE].

[22] J. Ambjørn and S. Wolfram, Properties of the vacuum. I. Mechanical and thermodynamic, Annals Phys. 147 (1983) 1 [INSPIRE].

[23] K. Kirsten, Casimir effect at finite temperature, J. Phys. A 24 (1991) 3281 [InSPIRE].

[24] K. Kirsten, Grand thermodynamic potential in a static space-time with boundary, Class. Quant. Grav. 8 (1991) 2239 [INSPIRE].

[25] J. Feinberg, A. Mann and M. Revzen, Casimir effect: the classical limit, Annals Phys. 288 (2001) 103 [hep-th/9908149] [INSPIRE].

[26] A. Scardicchio and R.L. Jaffe, Casimir effects: an optical approach. II. Local observables and thermal corrections, Nucl. Phys. B 743 (2006) 249 [quant-ph/0507042] [INSPIRE]. 
[27] S.C. Lim and L.P. Teo, Finite temperature Casimir energy in closed rectangular cavities: a rigorous derivation based on zeta function technique, J. Phys. A 40 (2007) 11645 [arXiv: 0804.3916] [INSPIRE].

[28] B. Geyer, G.L. Klimchitskaya and V.M. Mostepanenko, Thermal Casimir effect in ideal metal rectangular boxes, Eur. Phys. J. C 57 (2008) 823 [arXiv:0808.3754] [InSPIRE].

[29] A. Weber and H. Gies, Interplay between geometry and temperature for inclined Casimir plates, Phys. Rev. D 80 (2009) 065033 [arXiv:0906.2313] [InSPIRE].

[30] L.P. Teo, Finite temperature Cassimir effect for massive scalar field in spacetime with extra dimensions, JHEP 06 (2009) 076 [arXiv: 0903.3765] [INSPIRE].

[31] L.S. Brown and G.J. Maclay, Vacuum stress between conducting plates: an image solution, Phys. Rev. 184 (1969) 1272 [InSPIRE].

[32] N.D. Birrel and P.C.W. Davies, Quantum fields in curved space, Cambridge University Press, Cambridge U.K. (1982).

[33] S.A. Fulling, Aspects of quantum field theory in curved space-time, Cambridge University Press, Cambridge U.K. (1989).

[34] V.A. De Lorenci, L.G. Gomes and E.S. Moreira Jr., Hot scalar radiation setting bounds on the curvature coupling parameter, to appear in Class. Quant. Grav. [arXiv:1304.6041] [INSPIRE].

[35] S.A. Fulling and S.N.M. Ruijsenaars, Temperature, periodicity and horizons, Phys. Rept. 152 (1987) 135.

[36] C.T. Hill, One loop operator matrix elements in the Unruh vacuum, Nucl. Phys. B 277 (1986) 547 [inSPIRE].

[37] I.S. Gradshteyn and I.M. Ryzhik, Table of integrals, series, and products, Academic Press, U.S.A. (2007).

[38] E.R. Bezerra de Mello and A.A. Saharian, Vacuum polarization by a flat boundary in cosmic string spacetime, Class. Quant. Grav. 28 (2011) 145008 [arXiv:1103.2550] [INSPIRE].

[39] A.O. Sushkov, W.J. Kim, D.A.R. Dalvit and S.K. Lamoreaux, Observation of the thermal Casimir force, Nature Phys. 7 (2011) 230 [arXiv: 1011.5219] [INSPIRE].

[40] G. Arfken, Mathematical methods for physicists, Academic Press, U.S.A. (1985). 